\title{
Investigation of multidrug-resistant fatal colisepticaemia in weanling pigs
}

\author{
Authors: \\ Folorunso O. Fasina ${ }^{1}$ \\ Dauda G. Bwala ${ }^{1}$ \\ Evelyn Madoroba²

\section{Affiliations:} \\ ${ }^{1}$ Department of Production \\ Animal Studies, University of \\ Pretoria, South Africa \\ ${ }^{2}$ Bacteriology Section, \\ Agricultural Research \\ Council-Onderstepoort \\ Veterinary Institute, \\ South Africa

\section{Correspondence to:} \\ Folorunso Fasina \\ Email: \\ dayo.fasina@up.ac.za \\ Postal address: \\ Private bag X04, \\ Onderstepoort 0110, South \\ Africa \\ Dates: \\ Received: 08 May 2015 \\ Accepted: 27 Aug. 2015 \\ Published: 27 Nov. 2015 \\ How to cite this article: \\ Fasina, F.O., Bwala, \\ D.G. \& Madoroba, E., \\ 2015, 'Investigation of \\ multidrug-resistant fatal \\ colisepticaemia in weanling \\ pigs', Onderstepoort Journal \\ of Veterinary Research 82(1) \\ Art. \#986, 6 pages. http:// \\ dx.doi.org/10.4102/ojvr. \\ v82i1.986

\section{Copyright:} \\ C 2015. The Authors. \\ Licensee: AOSIS \\ OpenJournals. This work is \\ licensed under the Creative \\ Commons Attribution \\ License.
}

\section{Read online:}

Escherichia coli is usually a benign commensal of the gut microflora. However, when E. coli acquires virulence genes it can multiply rapidly and cause disease through colonisation of the intestinal mucosa. Escherichia coli can become a significant pathogen in young pigs. We report an investigation of fatal colisepticaemia in weanling pigs from emerging farms where piglets and weaners were diarrhoeic and the mortality rate ranged between $15 \%$ and $70 \%$ in each litter. Faecal and tissue samples were processed for histopathology, bacteriology and molecular biology (multiplex and monoplex polymerase chain reaction) and we recovered enteroaggregative multidrug-resistant $E$. coli producing EAST-1 enterotoxin. An association between poor housing conditions and the observed cases was established and future management programmes were recommended to reduce the impact of such pathogens. Enteroaggregative E. coli is becoming a major problem in the pig industry. It therefore becomes necessary to establish the full impact of E. coli on the South African pig industry and to determine the geographic extent of the problem.

\section{Communication}

Rotavirus, Escherichia coli, Clostridium perfringens, Isospora suis, transmissible gastro-enteritis virus and Enterococcus durans are amongst the most common diarrhoea-causing pathogens in piglets and are sometimes associated with neonatal and weanling deaths (Johnson et al. 1992; Martins et al. 2000; Vu-Khac, Holoda \& Pilipcinec 2004). Escherichia coli is generally a benign commensal of the gut microflora. However, when the bacterium acquires virulence genes it can multiply rapidly and colonise the intestinal mucosa by using surface proteins (fimbriae). The subsequent production of heat-stable or heat-labile toxins causes disease (Parma et al. 2000). Diarrhoea caused by E. coli affects all categories of young pigs (piglets, weaners and growers) to different degrees (Henton \& Engelbrecht 1997; Nagy \& Fekete 1999; Vu-Khac et al. 2006).

Recent reports have indicated that prevalence and isolation of antimicrobial-resistant E. coli are on the increase (Enne et al. 2008; Luppi et al. 2015; Toledo et al. 2012). Post-weaning diarrhoea (PWD) is a recurrent problem in weaned pigs (3-4 weeks of age) and previous studies have associated this condition with the F4 (mainly K88ac) and F18 fimbriae virulence factors (Nagy \& Fekete 1999; Vu-Khac et al. 2006). In South Africa specifically, Henton and Engelbrecht (1997) have serotyped 674 isolates from pigs and found that types O149, O141, O9, O20, 08 and a few others are prevalent in South Africa (arranged here in descending order of prevalence). F4 was associated with $46.9 \%$ of the isolates. Similar reports have been obtained from other smallholder farms and emerging pig farms despite slight variations in the pattern of presentation.

In December 2013, carcasses of two five-week-old Large White-Landrace cross weanlings were presented to the Pathology Laboratory of the Faculty of Veterinary Science, University of Pretoria (accession number S04444-13). Earlier, a report based on routine visits of the Porcine Herd Health team to the farm of origin (a start-up farm with 60 sow units) indicated that certain design and management errors had predisposed piglets in the farrowing unit to wet floors, cold draughts and hypothermia and the associated consequences. Suggestions for correction were offered. By the time the carcasses were presented for post-mortem examination, 14 sows had produced an average of 12 piglets per litter, of which 11 per litter were weaned at 4 weeks (weaning weight $\approx$ $6.7 \mathrm{~kg} ; n=151)$. PWD and mortality started 3-7 days post weaning and continued for the next 2 weeks, ultimately becoming self-limiting. Approximately $80 \%$ of the piglets experienced PWD to some extent. The mortality rate varied from $15 \%$ to $70 \%$ in affected litters.

Necropsy revealed severe generalised congestion, severe segmental catarrhal enteritis, moderate nephrosis and mild acute hepatosis. Generalised lymphadenomegaly due to cortical hyperplasia and diffuse moderate interstitial pneumonia with atelectasis were also observed. 
Multifocal villus crypts filled with a monopopulation of small bacterial rods histologically typical of $E$. coli were observed in tissue samples collected for histopathology (Figure 1a-c). The rod-like organisms adhered to enterocyte brush borders and were scattered within the lamina propria, extending to the lamina muscularis of the small intestine. The intestinal mucosa was not well differentiated and goblet cells were sparse, with a high mitotic rate (Figure 1d), indicative of regeneration and repair.

Multifocal atelectasis was observed in the lungs, interspersed by areas of marked interstitial pneumonia. Large numbers of mononuclear cells, mainly macrophages and lymphocytes, had infiltrated and distended the alveolar walls (Figure 1e-h). Multifocal areas of protein-rich alveolar oedema were observed. The lymph nodes showed numerous lymphoblasts within follicles, with prominent medullary congestion.

Based on the presumptive aetiological diagnosis, diarrhoeic faeces were collected from sick piglets during a follow-up visit to the farm. Culture on blood agar revealed both rough and smooth non-haemolytic E. coli organisms (pathology laboratory accession number B04025-14). During an invitro antimicrobial sensitivity evaluation, these organisms were found to be resistant to ampicillin, kanamycin, trimethoprim-sulphadimidine, oxytetracycline and tylosin, but susceptible to cetriofur, enrofloxacin and florfenicol. Subsequent faecal samples collected between January 2014 and June 2015 from this farm and others with matching production statuses revealed similar patterns of clinical signs, pathology and bacterial cultures. However, variable patterns of antibiotic resistance were observed, based primarily on the predominant antibiotics used on the farms.

All isolates were subcultured on blood agar and molecular characterisation was conducted. For this purpose, we conducted an investigation into the virulence factors associated with PWD using standardised protocols for polymerase chain reaction (PCR) and previously determined primers (Table 1).
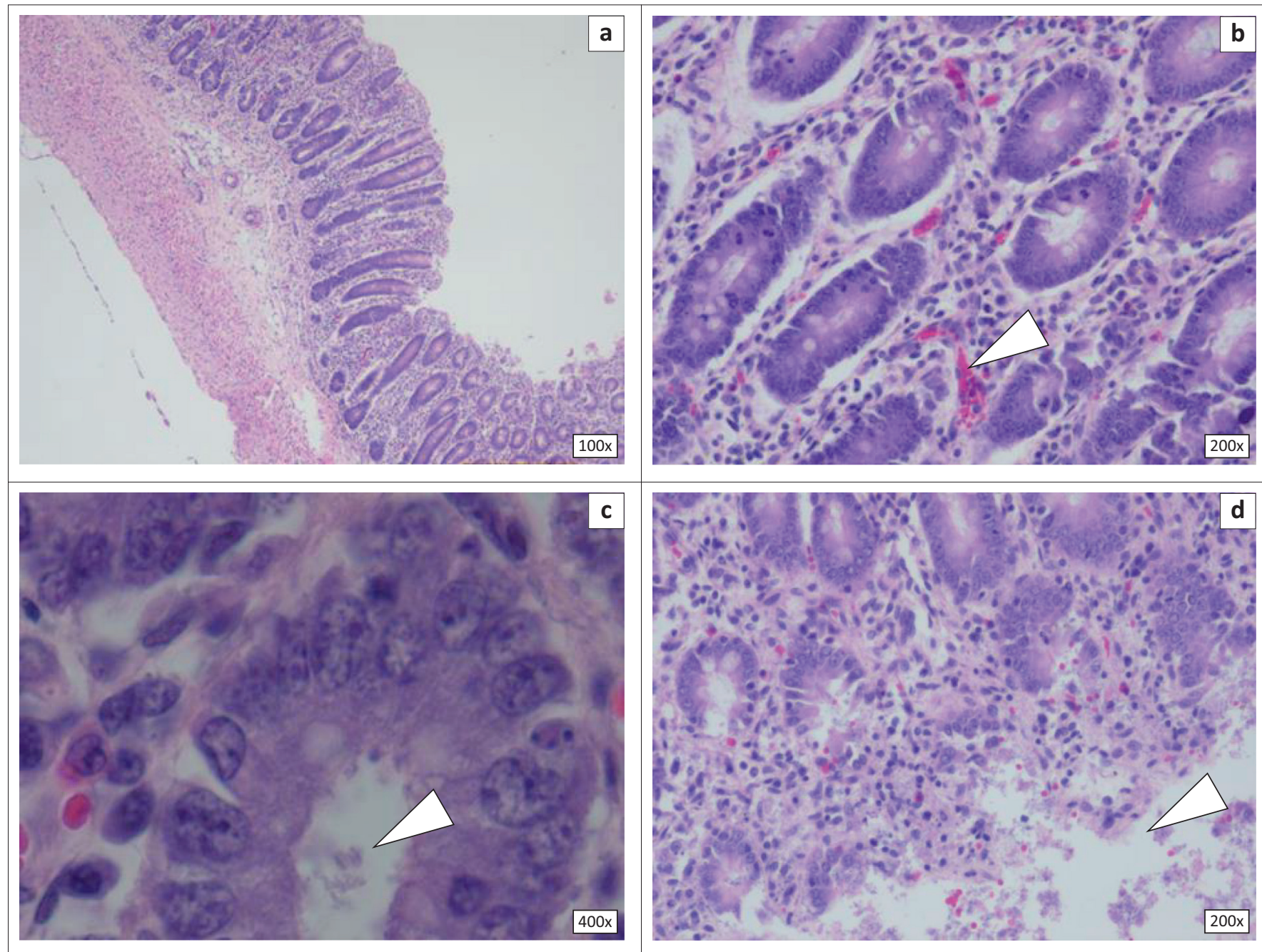

Source: D. Janse van Rensburg and J.C.A. Steyl, Section of Pathology, Faculty of Veterinary Science University of Pretoria

FIGURE 1: Histopathology of the intestinal and respiratory system tissue from the studied animals, stained with haematoxylin-eosin. (a) Overview of a transverse section of intestine; (b) Intestinal lumen, with normal lymphocytes and plasma cells in the lamina, mild congestion of enterocytes and lymphoid cells (arrow) and normal mucosal cells; (c) Lumen filled with bacteria and debris (arrow), normal enterocytes and goblet cells; (d) Section of the intestine, showing normal enterocytes, congestion, mild haemorrhage and autolytic cells (arrow); (e) Transverse section of the pig trachea showing the respiratory epithelium, blood vessels and hyaline cartilage; (f) Interstitial alveolar wall thickening (arrow); (g) Interstitial pneumonia and congestion; (h) Consolidated lung tissue with numerous bronchioles and congestion. 

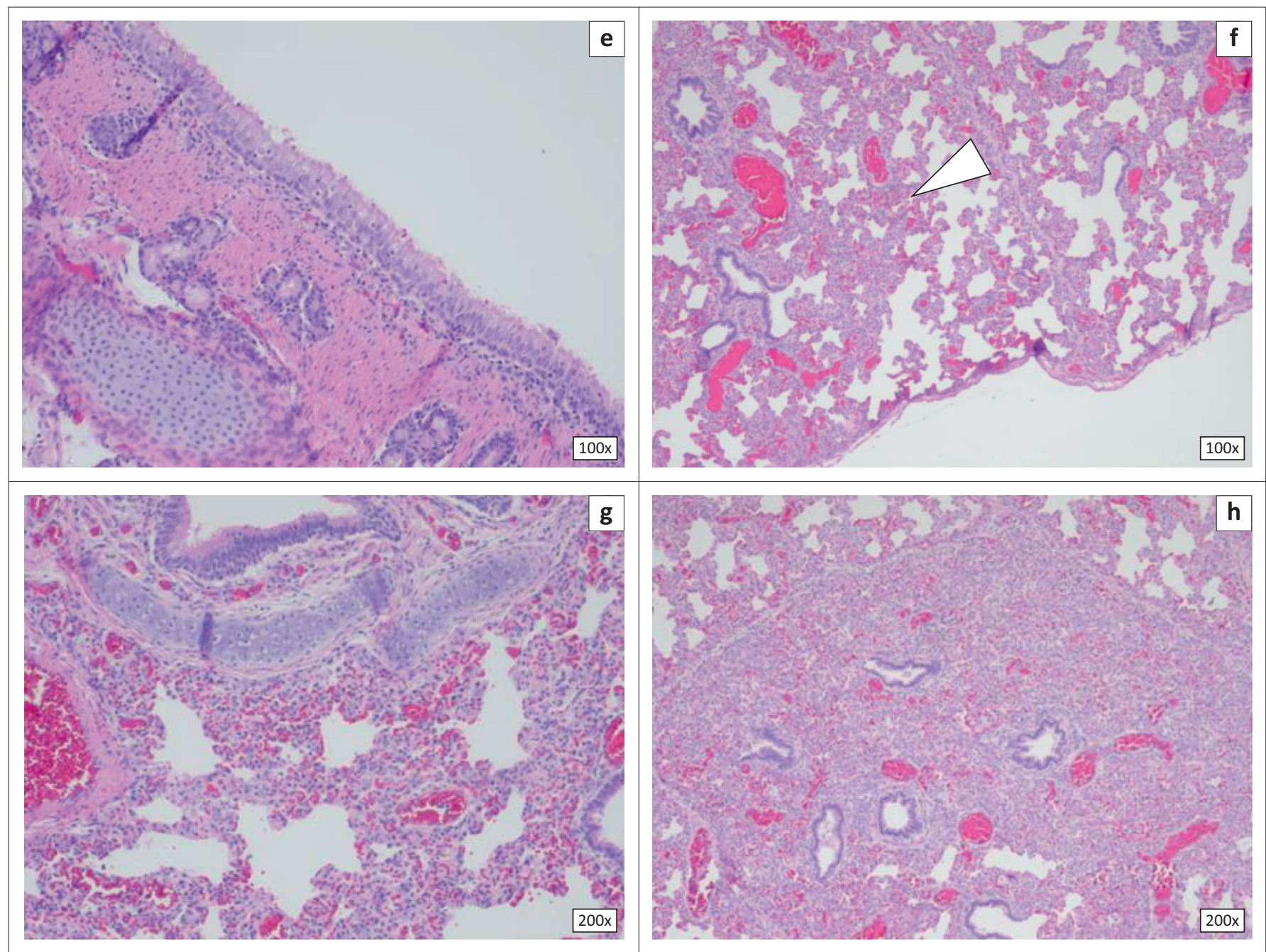

Source: D. Janse van Rensburg and J.C.A. Steyl, Section of Pathology, Faculty of Veterinary Science University of Pretoria

FIGURE 1 (Continues...): Histopathology of the intestinal and respiratory system tissue from the studied animals, stained with haematoxylin-eosin. (a) Overview of a transverse section of intestine; (b) Intestinal lumen, with normal lymphocytes and plasma cells in the lamina, mild congestion of enterocytes and lymphoid cells (arrow) and normal mucosal cells; (c) Lumen filled with bacteria and debris (arrow), normal enterocytes and goblet cells; (d) Section of the intestine, showing normal enterocytes, congestion, mild haemorrhage and autolytic cells (arrow); (e) Transverse section of the pig trachea showing the respiratory epithelium, blood vessels and hyaline cartilage; (f) Interstitial alveolar wall thickening (arrow); (g) Interstitial pneumonia and congestion; (h) Consolidated lung tissue with numerous bronchioles and congestion.

Escherichia coli DNA was extracted using cell lysis. Bacterial cells were boiled at $99{ }^{\circ} \mathrm{C}$ for $15 \mathrm{~min}$, followed by centrifugation. The supernatant containing crude DNA extracts was used in multiplex and monoplex PCR reactions targeting the following virulence factors: heat-labile toxin $(L T)$, heat-stable toxin A $(S T a)$, heat-stable toxin B $(S T b)$, shiga toxins Stx1, Stx2 and Stx2e, enteroaggregative heat-stable enterotoxin (EAST-1), adhesin involved in diffuse adherence 1 (AIDA-1), porcine attaching- and effacing-associated factor $(P A A)$ and fimbriae F4, F5, F6, F41 and F18. Primers used in the multiplex and monoplex PCR reactions were combined as described by Mohlatlole et al. (2013). The primers that were used for DNA amplification in the PCR reactions are listed in Table 1. The composition of the $25-\mu \mathrm{L}$ PCR reactions was as follows: $2.5 \mu \mathrm{M}$ of each primers, $5 \mu \mathrm{L}$ crude DNA extract, 12.5 $\mu \mathrm{L}$ PCR Master Mix (Fermentas) and sterile DNAse-free water (Fermentas). The conditions for amplification were as follows: $10 \mathrm{~min}$ initial denaturation at $94{ }^{\circ} \mathrm{C}$, followed by 30 cycles of denaturation at $94{ }^{\circ} \mathrm{C}$ for $30 \mathrm{~s}$, annealing at $56{ }^{\circ} \mathrm{C}$ for $30 \mathrm{~s}$ and extension at $72{ }^{\circ} \mathrm{C}$ for $1 \mathrm{~min}$. To ensure complete amplification of the PCR products, the thermocycling conditions included $7 \mathrm{~min}$ of extension at $72{ }^{\circ} \mathrm{C}$.

Monoplex PCR reactions were set up to confirm EAST toxins in the samples. Similar PCR amplification conditions were used, except for the volume of water being adjusted to a final volume of $25 \mu \mathrm{L}$ using sterile DNAse-free water.

Reference and control samples were included for all PCR reactions. For this purpose, reference samples that are known to be positive for enterotoxins and fimbriae were obtained from the Culture Collection of the Bacteriology Section of the Agricultural Research Council - Onderstepoort Veterinary Institute (ARC-OVI). The strains included B41 (F5:F41:STa), 1883-1 (F18:STa), 1883-2 (F41:F5:STa), 1883-3 (F41:F5:F6:STa), 1883-4 (F4:F5:LT:STb), 1474 K12-K99 Pienk (F5:STa), 1474 K12-K99 Geel (F5:STa) and K99 (F5:STa). In addition, isolates that were known to be positive for EAST-1, PAA, AIDA-1 and E. coli attaching and effacing (EAE) factor reference 
TABLE 1: Primer sequences of Escherichia coli virulence factors tested in the study.

\begin{tabular}{|c|c|c|c|c|}
\hline Virulence factor & Nucleotide sequence $\left(5^{\prime}-3^{\prime}\right)$ & Size (bp) & Target gene & Reference \\
\hline Sta-F & GGG TTG GCA ATT TTT ATT TCT GTA & 183 & estl & Cai et al. 2003; Ngeleka et al. 2003 \\
\hline Sta-R & ATT ACA ACA AAG TTC ACA AGC AGT A & - & - & - \\
\hline STb-F & ATG TAA ATA CCT ACA ACG GGT GAT & 360 & estll & - \\
\hline STb-R & TAT TTG GGC GCC AAA GCA TGC TCC & - & - & - \\
\hline LT-F & TAG AGA CCG GTATTA CAG AAATCT GA & 282 & elt & Cai et al. 2003; Ngeleka et al. 2003 \\
\hline LT-R & TCA TCC CGA ATT CTG TTA TAT ATG TC & - & - & - \\
\hline EAST-1-F & TCG GAT GCC ATC AAC ACA GT & 125 & ast $A$ & Cheng et al. 2006 \\
\hline EAST-1-R & GTC GCG AGT GAC GGC TTT GTA G & - & - & - \\
\hline Stx1-F & ATT CGC TGA ATG TCATTC GCT & 664 & stxl & Cai et al. 2003 \\
\hline Stx1-R & ACG CTT CCC AGA ATT GCA TTA & - & - & - \\
\hline Stx $2-\mathrm{F}$ & GAA TGA AGA AGA TGT TTA TAG CGG & 281 & stxll & Cai et al. 2003 \\
\hline Stx $2-\mathrm{R}$ & GGT TAT GCC TCA GTC ATT ATT AA & - & - & - \\
\hline Stx2e-F & GAA TGA AGA AGA TGT TTA TAG CGG & 454 & stx2e & Cai et al. 2003 \\
\hline Stx2e-R & TTT TAT GGA ACG TAG GTA TTA CC & - & - & - \\
\hline AIDA-1-F & ACA GTA TCA TAT GGA GCC A & 585 & $\operatorname{aid} A$ & Cheng et al. 2006 \\
\hline AIDA-1-R & TGT GCG CCA GAA CTA TTA & - & - & - \\
\hline EAE-F & CAT TAT GGA ACG GCA GAG GT & 790 & eae & Cheng et al. 2006 \\
\hline EAE-R & ATC TTC TGC GTA CTG CGT TCA & - & - & - \\
\hline PAA-F & ATG AGG AAC ATA ATG GCA GG & 360 & paa & Cheng et al. 2006 \\
\hline PAA-R & TCT GGT CAG GTC GTC AAT AC & - & - & - \\
\hline F4 (K88)-F & GAT GAA AAA GAC TCT GAT TGC A & 841 & faeG & Cai et al. 2003; Ngeleka et al. 2003 \\
\hline F4 (K88)-R & GAT TGC TAC GTT CAG CGG AGC G & - & - & - \\
\hline F5 (K99)-F & CTG AAA AAA ACA CTG CTA GCT ATT & 543 & fanA & Cai et al. 2003; Ngeleka et al. 2003 \\
\hline F5 (K99)-R & CAT ATA AGT GAC TAA GAA GGA TGC & - & - & - \\
\hline F41-F & GAT GAA AAA GAC TCT GAT TGC A & 682 & fim41a & Cai et al. 2003; Ngeleka et al. 2003 \\
\hline F41-R & TCT GAG GTC ATC CCA ATT GTG G & - & - & - \\
\hline F6 (987P)-F & GTT ACT GCC AGT CTA TGC CAA GTG & 463 & fasA & Cai et al. 2003; Ngeleka et al. 2003 \\
\hline F6 (987P)-R & TCG GTG TAC CTG CTG AAC GAA TAG & - & - & - \\
\hline $\mathrm{F} 18-\mathrm{F}$ & ATG AAA AGA CTA GTG TTT ATT TCT T & 513 & fedA & Ngeleka et al. 2003 \\
\hline
\end{tabular}

Note: Please see the full reference list of the article, Fasina, F.O., Bwala, D.G. \& Madoroba, E., 2015, 'Investigation of multidrug-resistant fatal colisepticaemia in weanling pigs', Onderstepoort Journal of Veterinary Research 82(1), Art. \#986, 6 pages. http://dx.doi.org/10.4102/ojvr.v82i1.986, for more information. $\mathrm{bp}$, base pair.

samples were included. E. coli ATCC 25922 was used as the negative control for virulence factors.

The PCR results indicated that of the 15 virulence factors tested, only EAST-1 yielded a positive band. Although most outbreaks of $E$. coli-derived diarrhoea in animals have been associated with strains with more than one virulence factor, in this case only EAST-1 toxin was observed (both E. coli isolates were positive for the 125-base-pair toxin; see Figure 2). EAST toxins, produced by enteroaggregative E. coli, are antigenically related to the heat-stable toxins produced by enterotoxigenic E. coli and are known to induce fluid secretion (Veilleux \& Dubreuil 2006). Nevertheless, the products encoded by EAST have also been found to be both diarrhoeagenic and non-pathogenic (Ruan et al. 2012). Although our results confirmed that E. coli was involved in causing disease and the associated mortalities in this case, involvement of other organisms was not explored. The EAST-1 toxins have been found in South African pigs in a previous study (Mohlatlole et al. 2013), which reported that $22.5 \%$ of the E. coli isolates carried the EAST-1 gene. Similar results have been obtained in studies from Korea and the Czech Republic, although higher prevalences were obtained in sucklers compared with weaner pigs (Lee et al. 2008; Zajacova, Konstantinova \& Alexa 2012).
As the EAST-1 toxin could exacerbate the pathogenesis of E. coli diarrhoea in weaned pigs (Choi et al. 2001), it will be necessary to determine the spatio-temporal extent of these organisms in the South African pig population to understand the epidemiology of the infection in the country. Furthermore, no E. coli vaccine available in South Africa confers protection against the EAST-1 toxin, which may partially explain the high mortality observed in some of the cases.

Based on the outcome of the pathology and PCR results, a conclusive diagnosis of colisepticaemia was made. At weaning, piglets are subjected to a variety of stress factors, including the withdrawal of the dam and sow's milk, a dip in the concentration of $\mathrm{CD} 4^{+}$and $\mathrm{CD} 8^{+}$cells, neutrophils and lymphocytes, and a post-weaning syndrome that consists of PWD, oedema disease and endotoxin shock (Almond \& Kirk 2010; Van Beers-Schreurs et al. 1992). The change in gut microflora associated with the changing diet around the time of weaning may lead to $E$. coli overgrowth and subsequent enteritis and endotoxaemia. On this farm, several cases of diarrhoea were observed in the farrowing and weaner pens and respiratory distress was observed in young weaners. Although the E. coli-associated enteritis could have compromised the integrity of the intestinal villi and 


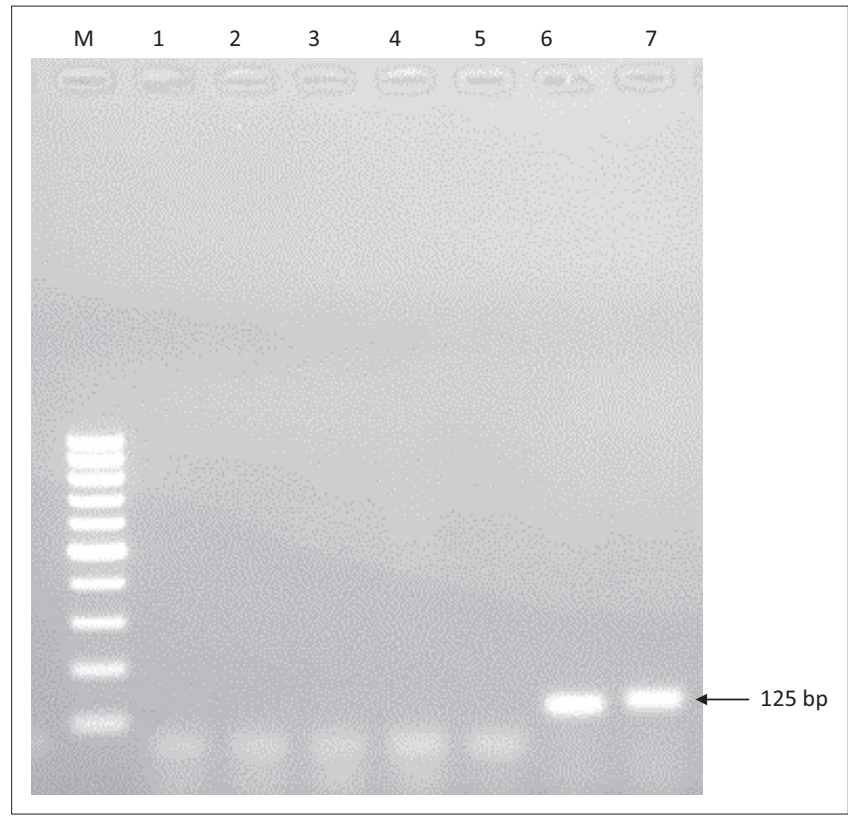

Source: E. Madoroba, Bacteriology Section, ARC-OVI

EAST, enteroaggregative heat-stable enterotoxin; LT, heat-labile toxin; STa, heat-stable toxin A; STb, heat-stable toxin B.

FIGURE 2: Gel electrophoresis showing EAST-1 positive amplicons. Lanes: 1 100-bp plus DNA ladder; 2, STb negative; 3, LT negative; 4, E. coli ATCC 25922 negative control strain; 5 , STa negative; 6 and 7 , EAST positive.

reduced the efficiency of feed utilisation, the cause of death in these weaner pigs was E. coli-associated endotoxaemia. No cases of piglet anaemia were observed in the herd and the oxygen-carrying capacity of haemoglobin did not appear compromised. The consolidation and interstitial alveolar wall thickening observed in the lungs notably reduced the capacity of the lungs in complying with their primary role of oxygen-carbon dioxide exchange at the alveolar level. Fast-growing weaner pigs need an increasing level of such gaseous exchange to meet their physiologic needs at this stage. It becomes vitally important to reduce other associated complications (e.g. digestive or respiratory) arising from E. coli infection at the time of weaning.

We recommended changes in management protocol at this farm to ensure dry floors and hygiene in the farrowing and weaner units. Establishment of a vaccination protocol that includes routine E. coli vaccination of sows 2 weeks before parturition is also recommended to boost colostral immunity and decrease the magnitude of antibiotic treatment in subsequent outbreaks.

\section{Acknowledgements}

We thank the staff of the Pathology Section of the Department of Paraclinical Sciences, the Microbiology Laboratory of the Department of Veterinary Tropical Diseases and the Porcine Herd Health students (PHP 650) of the Faculty of Veterinary Science, University of Pretoria, who all participated in the investigation. We also appreciate the cooperation of the farmer. The molecular biology work was carried out at the ARC-OVI, Onderstepoort, South Africa.

\section{Competing interests}

The authors declare that they have no financial or personal relationships that may have inappropriately influenced them in writing this article.

\section{Authors' contributions}

F.O.F. (University of Pretoria) and D.G.B. (University of Pretoria) drafted and revised the manuscript. F.O.F. attended to the case and performed clinical sampling and follow-up. E.M. (ARC-OVI) was responsible for bacteriology work. All authors contributed to the writing of the manuscript and approved it for submission.

\section{References}

Almond, G.W. \& Kick, A.R., 2010, Effects of stress on immune cell populations in pigs, final report, North Carolina Pork Council, Raleigh.

Cai, H.Y., Archambault, M., Gyles, C.L. \& Prescott, J.F., 2003, 'Molecular genetic methods in the veterinary clinical bacteriology laboratory: Current usage and future applications', Animal Health Research Reviews 4(2), 73-94. PMID: and future 15134292.

Cheng, D., Sun, H., Xu, J. \& Gao, S., 2006, 'PCR detection of virulence factor genes in Escherichia coli isolates from weaned piglets with edema disease and/or diarrhea in China', Veterinary Microbiology 115(4), 320-328. PMID: 16567064, http:// dx.doi.org/10.1016/j.vetmic.2006.02.013

Choi, C., Cho, W., Chung, H., Jung, T., Kim, J. \& Chae, C., 2001, 'Prevalence of the enteroaggregative Escherichia coli heat-stable enterotoxin 1 (EAST-1) gene in isolates in weaned pigs with diarrhoea and/or edema disease' Veterinary Microbiology 81(1), 65-71. http://dx.doi.org/10.1016/S0378-1135(01) 00332-7

Enne, V.I., Cassar, C., Sprigings, K., Woodward, M.J. \& Bennett, P.M., 2008, 'A high prevalence of antimicrobial resistant Escherichia coli isolated from pigs and a low prevalence of antimicrobial resistant $E$. coli from cattle and sheep in Great Britain at slaughter', FEMS Microbiology Letters 278, 193-199. PMID: 18053066.

Henton, M.M. \& Engelbrecht, M.M., 1997, 'Escherichia coli serotypes in pigs in South Africa', Onderstepoort Journal of Veterinary Research 64, 175-187. PMID: 9467172.

Johnson, W.M., Fitzgerald, G.R., Welter, M.W. \& Welter, C.J., 1992, 'The six most common pathogens responsible for diarrhoea in newborn pigs', Veterinary Medicine 4, 382-386.

Lee, S.I., Kang, S.G., Kang, M.L. \& Yoo, H.S., 2008, 'Development of multiplex polymerase chain reaction assays for detecting enterotoxigenic Escherichia coli and their application to field isolates from piglets with diarrhoea', Journal of Veterinary Diagnostic Investigation 20, 492-496. PMID: 18599856, http://dx.doi. org/10.1177/104063870802000413

Luppi, A., Bonilauri, P., Dottori, M., Gherpelli, Y., Biasi, G., Merialdi, G. et al., 2015, 'Antimicrobial resistance of F4+ Escherichia coli isolated from swine in Italy', Transboundary and Emerging Diseases 62(1), 67-71. PMID: 23906344, http:// dx.doi.org/10.1111/tbed.12081

Martins, M.D.F., Martínez-Rossi, N.M., Ferreira, A., Brocchi, M., Yano, T., Castro, A.F.P. et al., 2000, 'Pathogenic characteristics of Escherichia coli strains isolated from newborn piglets with diarrhoea in Brazil', Veterinary Microbiology 76, 51-59. PMID: 10925041, http://dx.doi.org/10.1016/S0378-1135(00)00223-6

Mohlatlole, R.P., Madoroba, E., Muchadeyi, F.C., Chimonyo, M., Kanengoni, A.T. \& Dzomba, E.F., 2013, 'Virulence profiles of enterotoxigenic, shiga toxin and enteroaggregative Escherichia coli in South African pigs', Tropical Animal Health and Production 45(6), 1399-1405. PMID: 23417826, http://dx.doi.org/10.1007/ s11250-013-0377-4

Nagy, B. \& Fekete, P.Z., 1999, 'Enterotoxigenic E. coli (ETEC) in farm animals', Veterinary Research 30, 259-284. PMID: 10367358.

Ngeleka, M., Pritchard, J., Appleyard, G., Middleton, D.M. \& Fairbrother, J.M., 2003, 'Isolation and association of Escherichia coli AIDA-1/STb, rather than EAST-1 pathotype, with diarrhea in piglets and antibiotic sensitivity of isolates', Journal of Veterinary Diagnostic Investigation 15(3), 242-252. PMID: 12735346, http:// of Veterinary Diagnostic Investigation 15(3),
$\mathrm{dx}$. doi.org/10.1177/104063870301500305

Parma, A.E., Sanz, M.E., Vinas, M.R., Cicuta, M.E., Blanco, J.E., Boehringer, S.I. et al., 2000, 'Toxigenic Escherichia coli isolated from pigs in Argentina', Veterinary Microbiology 72, 269-276. PMID: 10727836, http://dx.doi.org/10.1016/S03781135(99)00167-4

Ruan, X., Crupper, S.S., Schultz, B.D., Robertson, D.C. \& Zhang, W., 2012, 'Escherichia coli expressing EAST1 toxin did not cause an increase of cAMP or cGMP levels in cells, and no diarrhea in 5-day old gnotobiotic pigs', PLOS ONE 7(8), e43203. PMID: 22905235, http://dx.doi.org/ 10.1371/journal.pone.0043203

Toledo, A., Gomez, D., Cruz, C., Carreon, R., Lopez, J., Giono, S. et al., 2012, 'Prevalence of virulence genes in Escherichia coli strains isolated from piglets in the sucklin and weaning period in Mexico', Journal of Medical Microbiology 61, 148-156. PMID: 21852524, http://dx.doi.org/10.1099/jmm.0.031302-0 
Van Beers-Schreurs, H.M., Vellenga, L., Wensing, T. \& Breukink, H.J., 1992, 'The pathogenesis of the post-weaning syndrome in weaned piglets: A review',
Veterinary Quarterly 14(1), 29-34. PMID: 1574833, http://dx.doi.org/10.1080/0 Veterinary Quarterly 14(1)
1652176.1992.9694322

Veilleux, S. \& Dubreuil, D.J., 2006, 'Presence of Escherichia coli carrying the EAST-1 toxin gene in farm animals', Veterinary Research 37, 3-13. PMID: 16336921.

Vu-Khac, H., Holoda, E. \& Pilipcinec, E., 2004, 'Distribution of virulence genes in Escherichia coli strains isolated from diarrhoeic piglets in the Slovak Republic' Journal of Veterinary Medicine Series B 51, 343-347. PMID: 15525362.
Vu-Khac, H., Holoda, E., Pilipcinec, E., Blanco, M., Blanco, J.E., Mora, A. et al., 2006, 'Serotypes, virulence genes, and PFGE profiles of Escherichia coli isolated from pigs with post weaning diarrhoea in Slovakia', BMC Veterinary Research 2, 10 PMID: 16549022, http://dx.doi.org/10.1186/1746-6148-2-10

Zajacova, Z.S., Konstantinova, L. \& Alexa, P., 2012, 'Detection of virulence factors of Escherichia coli focused on prevalence of EAST-1 toxin in stool of diarrheic and non-diarrheic piglets and presence of adhesion involving virulence factors in astA positive strains', Veterinary Microbiology 154, 369-375. PMID: 21864997, http:// dx.doi.org/10.1016/j.vetmic.2011.07.029 